\title{
Gelebte Vielfalt in der Hochschule durch eine diversitätsorientierte Zugangspraxis
}

Anmerkungen zur sozialen Dimension der österreichischen Hochschulpolitik

Durchlässigkeit, Diversitätsorientierung und Gerechtigkeit sind Schlagworte, die gegenwärtig in hochschulpolitischen Diskursen Konjunktur haben. Sie werden eingesetzt, um die "soziale Dimension" des Hochschulsystems stärker in den Blickpunkt zu rücken. Das Ziel lautet: Der Zugang zum tertiären Bildungssektor soll für die bislang unterrepräsentierten Studierendengruppen durch die Anerkennung von nicht-formalen und informellen Bildungswegen „integrativer" werden. Diese Entwicklungen könnten einen wesentlichen Schritt in Richtung Inklusion bedeuten, sind aber aus Sicht der Sozialpädagogik mit einigen Herausforderungen verbunden, die es kritisch zu begleiten und zu beleuchten gilt.

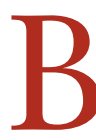
ildung ist nicht nur in der deutschsprachigen (Sozial-)Pädagogik ${ }^{1}$ ein grundlegender Begriff, sondern mittlerweile auch in vielen anderen (Sub-) Disziplinen, wie etwa der (Bildungs-)Soziologie und der (Bildungs-)Ökonomie, ein wesentlicher Gegenstand von wissenschaftlichen Auseinandersetzungen. Eines

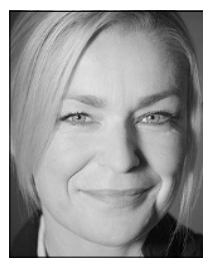

Marina Tomic Hensel

St. Pölten, Österreich

*1979, Studium Soziale Arbeit (Diplom), Studium Gender Studies (MA), Studium Bildungswissenschaft (Doktoratsstudium laufend) Marina.Tomic-Hensel@fhstp.ac.at

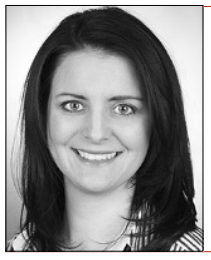

Patricia Renner

St. Pölten, Österreich

*1982, Sozialpädagogin, Studium Soziale Arbeit (BA, MA)

Patricia.Renner@fhstp.ac.at

Zusammenfassung Den Zugang zu Hochschulbildung sozial integrativer und die Teilhabe diversitätssensibel zu gestalten, ist gegenwärtig eines der zentralen Ziele in der österreichischen Hochschulpolitik. Der Beitrag geht der Frage nach, wie eine an der Diversität orientierte Zugangspraxis zur Inklusion an Hochschulen beitragen kann und welche Aufgabe dabei der Sozialpädagogik zukommt.

Schlüsselwörter Hochschulzugang, Inklusion, Diversität der zentralen Themen der unter „Bildungsforschung“ zusammengefassten interdisziplinären Perspektiven ist die ungleiche Verteilung bzw. die „soziale Vererbung“ von Bildungschancen (vgl. Tippelt und Schmidt 2009, S. 10). Ein Befund aus der Hochschulforschung ist, dass sich im Zuge der Expansion des Hochschulsystems die Reproduktion sozialer Ungleichheit beim Zugang zum Bildungswesen bis heute nicht wesentlich verringert hat (vgl. Wolter 2013). So konstatiert Wolter für Europa: „In almost all countries growth in participation has not been accompanied by a process of social inclusion or only by a very modest process of social opening. “ (ebd., S. 218). Dies belegen auch aktuelle Zahlen für Österreich. So ist „die Studierwahrscheinlichkeit für Studierende aus ,bildungsnahen' Elternhäusern rund 2,38mal höher als für jene aus ,bildungsfernen'." (BMWFW 2017, S. 13, bezugnehmend auf die Studierenden-Sozialerhebung 2015) Der elterliche Bildungshintergrund ist jedoch nur einer der Faktoren, die sich auf den Zugang (und Verbleib) im tertiären Bildungssektor (negativ) auswirken können. Niedriger sozioökonomischer Status der Familie, Geschlecht, Migrationshintergrund, Behinderung und/ oder chronische Erkrankung sowie regionale Herkunft spielen bei der Verteilung der Zugangschancen nach wie vor eine Rolle (vgl. BMWFW 2017, S. 12 ff.). Unterrepräsentiert sind in der Hochschule ebenso Studierende, deren Bildungsbiografien von dem traditionellen (linearen und unmittelbar konsekutiven) Bildungsweg abwei- 
chen (HSK 2015, S. 13 ff.). Problematisch sind die ungleichen Chancen deshalb, weil der Zugang zu Bildung nach wie vor nicht nur für die gesellschaftliche und politische Teilhabe von Menschen (vgl. Kallhoff 2012), sondern auch für ihre Lebenschancen und die ihrer Kinder entscheidend ist (vgl. Marginson 2012, S. 6, bezugnehmend auf McMahon 2009). ${ }^{2}$

Insofern davon ausgegangen werden kann, dass die „sozialen Bedingungen der Bildung [...] und die Bildungsbedingungen des sozialen Lebens" (Natorp 1968, S. 9 , zit. in Hamburger 2012, S. 120) ein zentrales Thema von Sozialpädagogik darstellen (vgl. ebd., S. 120 f.), lohnt es sich aus unserer Perspektive den Blick auf die gegenwärtigen Entwicklungen in der österreichischen Hochschulpolitik zu lenken und einige Gedanken zu Chancen und Herausforderungen bei der Umsetzung $\mathrm{zu}$ formulieren. Untersuchungsgegenstand ist die „Nationale Strategie zur sozialen Dimension in der Hochschulbildung“ (BMWFW 2017) und hier insbesondere die Zielsetzung eines „integrativeren Zugangs“ für die im Hochschulsystem bisher unterrepräsentierten Studierendengruppen. Die leitende Frage ist: Wie kann eine an der Diversität orientierte Zugangspraxis zu einer „gelebten Vielfalt" werden?

\section{Die "soziale Dimension" in der Hochschulbildung}

Die „Nationale Strategie zur sozialen Dimension in der Hochschulbildung “ des Bundesministeriums für Bildung, Wissenschaft und Forschung ist „das erste gesamthafte Strategiedokument in der österreichischen Hochschulentwicklung “ zu den Themen Durchlässigkeit und Zugangsgerechtigkeit (BMWFW 2017, S. 7 f.). Das formulierte Ziel der Strategie ist es, „den Zugang zu Hochschulbildung sozial integrativer und die Teilhabe breiter und heterogenitäts- und diversitätssensibel zu gestalten “ (ebd., S. 8). Insbesondere richtet sich der Blick auf die unterrepräsentierten Gruppen, nicht-traditionelle Studierende sowie Gruppen mit spezifischen Anforderungen, wie z. B. Studierende mit Behinderung, mit Betreuungspflichten etc. - wobei die Gruppen nicht ohne Überschneidungen sind (vgl. ebd., S. 10 ff.). Legitimiert werden die strategischen Zielsetzungen mit der Notwendigkeit, den gesellschaftlichen und wirtschaftlichen Entwicklungen angemessen zu begegnen (vgl. ebd., S. 7).

Es werden drei Zieldimensionen formuliert, die jeweils drei Aktionslinien mit konkreten Maßnahmen enthalten. Die drei Zieldimensionen sind:

1. Integrativerer Zugang,

2. Abbruch verhindern, Studienerfolg steigern und

3. Rahmenbedingungen schaffen und hochschulpolitische Steuerung optimal einsetzen (vgl. BMWFW 2017, S. 20 ff.).
Wir möchten hier auf den Aspekt des „integrativeren Zugangs" durch Anerkennung und Validierung nichtformaler und informeller Kompetenzen näher eingehen. Als theoretisch-reflexive Hintergrundfolie dienen Perspektiven aus der sozialpädagogischen Bildungsforschung. Diese rücken Bildungsorte in den Fokus, in denen sich informelle und nicht-formale Bildungs- und Lernprozesse vollziehen (vgl. exemplarisch Haag 2009; Thole und Höblich 2008). Im Folgenden formulieren wir einige Gedanken dazu.

\section{„Die andere Seite der Bildung"}

Mit der Prämisse eines „erweiterten Bildungsverständnisses" wird in der sozialpädagogischen Bildungsforschung auf die Desiderate der traditionellen Bildungsforschung reagiert, die bislang vorwiegend die Schule und das Bildungssystem fokussiert (vgl. Haag 2009, S. 216). Auf die „andere Seite der Bildung “ zu blicken, ist mit dem Anspruch verbunden, „die Vielfalt der vor und neben der Schule liegenden Bildungspotenziale komplementär einzubeziehen und systematisch zu aktivieren." (Rauschenbach und Otto 2008, S. 5). Damit wird der Erkenntnis Rechnung getragen, dass sich Bildungs- und Lernprozesse nicht nur in institutionalisierten Settings, sondern auch ungeplant bzw. weniger geplant und in weniger eindeutigen Lernwelten und Bildungsorten vollziehen (können) (vgl. dazu auch Rauschenbach und Otto 2008, S. 5; zur Debatte s. a. Thole und Höblich 2008). Diese sind beispielsweise Familien, Freundschaften, Peers (informelle Bildungsorte) bzw. Vereine, Verbände und Institutionen der Kinder- und Jugendarbeit (nicht-formale Bildungsorte) (vgl. Thole und Höblich 2008).

In der „Nationalen Strategie zur sozialen Dimension in der Hochschulbildung “ wird Anerkennung eben dieser - weniger formalisierten - Bildungsprozesse als zentrale Voraussetzung für ein durchlässiges und sozial integratives Hochschulsystem betrachtet, auf deren Grundlage bildungspolitische Forderungen formuliert werden. Den Hochschulen kommt die Aufgabe zu, Zulassungsbedingungen zu modifizieren, sprich Verfahren zu entwickeln, um die informellen und nicht-formalen Bildungs- und Lernprozesse entsprechend abbilden zu können. Damit sind aus unserer Sicht einige Herausforderungen verbunden, die wir im Folgenden skizzieren möchten.

\section{Überlegungen zu einer diversitätsorientierten Zugangspraxis}

Unsere Eingangsfrage war, wie eine an Diversität orientierte Zugangspraxis zu einer „gelebten Vielfalt“ werden kann. Unser Verständnis von Diversität orientiert sich dabei an der Idee der Inklusion von gesellschaftlich be- 
nachteiligten Personengruppen. Das Thema bearbeiten wir auch in dem Bewusstsein, dass Selektivitätsmechanismen nicht erst in der Hochschule wirksam werden, sondern in gesellschaftlichen Ungleichheitsverhältnissen angelegt sind. In der sozialpädagogischen Bildungsforschung wird darauf verwiesen, dass die Chancen, sich auf informellen bzw. nicht-formalen Bildungswegen Wissen und Fähigkeiten anzueignen - analog zu den formalisierten Bildungsstrukturen - ungleich verteilt sind. So hängen etwa die Möglichkeiten der Freizeitgestaltung, der Nutzung von Medien sowie der Zugehörigkeit zu sozialen Netzwerken und Peer-Groups u. a. vom Geschlecht, der ethnischen und sozialen Herkunft sowie von den materiellen Ressourcen der Kinder und Jugendlichen bzw. deren Herkunftsfamilie ab (vgl. Thole und Höblich 2008, S. 71 ff., bezugnehmend auf diverse Studien). Insofern die Chancen auf ein Hochschulstudium bzw. einen Hochschulabschluss in hohem Maße durch die Bildungsbiografie davor bestimmt sind, könnte zwar die Anerkennung von nicht-formalen und informellen Bildungswegen einen wichtigen Schritt darstellen, um längerfristig den Effekt der Vererbung von Bildungschancen (und Lebenschancen) zu verringern. Kritisch zu reflektieren ist jedenfalls die Tatsache, dass die Disparitäten nicht erst bei der Zulassung zum Hochschulsystem sichtbar werden. Aus diesem Grund verweisen wir zunächst auf die „sozialen Bedingungen von Bildung“, die einem „integrativen Hochschulzugang “ aus unserer Sicht zugrunde liegen. Danach skizzieren wir Aspekte, die bei der Zulassung zur Hochschule und der Gestaltung des Studiums eine zentrale Rolle spielen könnten, um die Hochschule inklusiver zu gestalten.

\section{Bildungsprozesse ermöglichen}

Wenn die Anerkennung von nicht-formalen und informellen Bildungswegen zu mehr Inklusion beitragen soll, ist es zunächst Aufgabe der Politik, Bedingungen für förderliche Bildungsprozesse im Alltag zu schaffen, z. B. mit Maßnahmen der Jugend- und Familienpolitik (vgl. dazu auch Thiersch 2008, S. 979). Damit sind nicht nur informelle, sondern auch die nicht-formalen Bildungsprozesse angesprochen, die sich etwa in den Institutionen der Kinder- und Jugendarbeit vollziehen. In diesen werden Möglichkeiten eröffnet, „Kompetenzen zu erwerben, die andernorts in dieser Spezifität nicht ausgebildet werden können " (Thole und Höblich 2008, S. 83). Aus dieser Perspektive wird Sozialpädagogik zu einer wesentlichen Akteurin, wenn es darum geht, Bildungsprozesse bei jungen Menschen zu initiieren, zu erkennen und zu unterstützen (vgl. dazu auch Rauschenbach und Otto 2008, S. 23). Dabei ist das Ziel weniger, Beschäftigungsfähigkeit herzustellen, als viel- mehr gesellschaftliche Teilhabe und Inklusion zu fördern, im Sinne einer selbstbestimmten Lebensführung. Angesichts der aktuellen bildungspolitischen Diskussionen ist zunächst eine inhaltliche Debatte darüber zu führen, mit welchen Erwartungen der (neue) Bildungsauftrag verknüpft ist und welches sozialpädagogische Verständnis von Bildung diesem zugrunde liegen kann (vgl. dazu auch Rauschenbach und Otto 2008). Die Feststellung, dass es hierfür die entsprechenden materiellen, fachlichen und zeitlichen Ressourcen braucht, mag trivial klingen, ist aber in Zeiten der Ökonomisierung des Sozialen eine unumgängliche (vgl. dazu Tomic Hensel 2019).

\section{Bildungsprozesse anerkennen}

Entscheidend für eine inklusive Zulassungspraxis ist in weiterer Folge die Bereitschaft von Hochschulen, neue Wege zu gehen und qualitative Anerkennungsverfahren zu entwickeln, um die individuellen Bildungsprozesse bei der Zulassung abbilden zu können. ${ }^{3}$ Die Herausforderung liegt darin, transparente Modelle anzudenken, die es erlauben, Fähigkeiten, Wissen, Werte und Haltungen zu erfassen, die über die Leistungsdokumentation in Zertifikaten, Zeugnissen, etc. hinausgehen. Diese Prozesse implizieren die Beschäftigung mit der Frage nach dem Verständnis von nicht-formaler und informeller Bildung bzw. der Frage, was aus welchen Gründen als „Voraussetzung“ für das jeweilige Studium gelten kann und was nicht. Eine kritische Reflexion der Konzeption von Bildung ist auch deshalb essentiell, weil der Nutzen und die Funktion vom Studium in hochschulpolitischen Dokumenten vorwiegend in Bezug auf deren wirtschaftliche Bedeutung thematisiert werden. Die Gefahr der Vereinseitigung des Bildungsverständnisses als „brauchbare Kompetenz“ ist vor allem dann zu reflektieren, wenn informelle und nicht-formale Kompetenzen in formale Strukturen eingespeist werden sollen, wie dies in der „Nationalen Strategie zur sozialen Dimension in der Hochschulbildung “ als Auftrag an Hochschulen formuliert worden ist. Damit ist die Frage nach dem Verhältnis von informeller, nicht-formaler und formaler Bildungskonzeptionen berührt, die auch bei der Gestaltung des Studiums zu verhandeln ist.

\section{Bildungsprozesse fördern}

Non-formal und informell erworbene Fähigkeiten und Fertigkeiten sind nicht als Kompensation formeller Bildung zu verstehen. Vielmehr geht es darum, die Komplementarität von unterschiedlichen Bildungserfahrungen im Hochschulalltag anzuerkennen und abzubilden. Insofern das Studium von Sozialpädagogik an der Fachhochschule nicht nur wissenschaftlich fundiert und pra- 
xisbezogen sein soll ( $\mathbb{3}$ FHStG $)^{4}$, sondern die Persönlichkeitsentwicklung adressiert, gilt es auch hier jenes (Erfahrungs-)Wissen sowie Fähigkeiten in den Blick zu nehmen, die im Alltag erworben wurden. Dies ist umso bedeutender, als Empathie, moralische Urteilsfähigkeit, Frustrationstoleranz, konstruktiver Umgang mit Problemen, Selbständigkeit und Selbstmanagement insbesondere auch in alternativen Bildungsorten erworben bzw. gefördert werden (Thole und Höblich 2008, S. 76 ff.).

Die genannten Fähigkeiten sind sowohl im allgemeinen Hochschulalltag als auch in der Berufspraxis von Sozialpädagog_innen (und nicht nur in diesen) wesentlich. Vor dem Hintergrund der bisherigen Überlegungen stellt sich für die Gestaltung des Studiums der Sozialpädagogik mitunter die Frage, wie die individuellen Bildungserfahrungen didaktisch aufzugreifen sind und wie „alternative Bildungsorte“ in formalisierten Strukturen beibehalten bzw. geschaffen werden können. Studierende für die informellen Bildungsprozesse zu sensibilisieren ist auch deshalb wesentlich, weil sie sich in der Praxis mit dem eigenen Bildungsauftrag auseinandersetzen müssen.

\section{Resümee}

Um an der Diversität orientierte Zugänge zu Hochschulbildung zu ermöglichen und zu einer "gelebten Vielfalt“ zu machen, sind unserer Meinung nach nicht nur ein Wille zur Veränderung, sondern auch entsprechende Strukturen und Ressourcen notwendig - bei der Ermöglichung von informellen und nicht-formalen Bildungsprozessen, bei der Entwicklung von entsprechenden Zulassungsmodellen und in der Schaffung von Rahmenbedingungen ${ }^{5}$, die insbesondere den unterrepräsentierten Studierendengruppen einen Verbleib im Studium sowie einen Abschluss ermöglichen. Dies betrifft auch Fragen nach finanziellen Fördermöglichkeiten für Studierende, auch in nicht konsekutiven Studiengängen. Allem voran braucht es inhaltliche Auseinandersetzungen darüber, welche Bildungsprozesse in den Anerkennungsverfahren in den Blick kommen und welche nicht und wie sich diese Entscheidungsprozesse gestalten. Ob die neuen Zulassungsmodelle tatsächlich zu mehr Vielfalt und Inklusion beitragen, ist vor dem Hintergrund der Einbettung der Bildungschancen in gesamtgesellschaftliche Zusammenhänge bzw. der ungleichen sozialen Bildungsbedingungen und der Selektivität des Hochschulsystems jedenfalls kritisch zu reflektieren. Dies ist eine Aufgabe, die sozialpädagogische Bildungsforschung übernehmen könnte.
Funding. Open access funding provided by FH St. Pölten, University of Applied Sciences.

Open Access. Dieser Artikel wird unter der Creative Commons Namensnennung 4.0 International Lizenz veröffentlicht, welche die Nutzung, Vervielfältigung, Bearbeitung, Verbreitung und Wiedergabe in jeglichem Medium und Format erlaubt, sofern Sie den/die ursprünglichen Autor(en) und die Quelle ordnungsgemäß nennen, einen Link zur Creative Commons Lizenz beifügen und angeben, ob Änderungen vorgenommen wurden. Die in diesem Artikel enthaltenen Bilder und sonstiges Drittmaterial unterliegen ebenfalls der genannten Creative Commons Lizenz, sofern sich aus der Abbildungslegende nichts anderes ergibt. Sofern das betreffende Material nicht unter der genannten Creative Commons Lizenz steht und die betreffende Handlung nicht nach gesetzlichen Vorschriften erlaubt ist, ist für die oben aufgeführten Weiterverwendungen des Materials die Einwilligung des jeweiligen Rechteinhabers einzuholen. Weitere Details zur Lizenz entnehmen Sie bitte der Lizenzinformation auf http://creativecommons.org/licenses/by/4.0/deed.de.

1. In Anlehnung an Lothar Böhnisch verstehen wir Sozialpädagogik als eine „sozial- und erziehungswissenschaftliche Disziplin im allgemeinen Sinne“ und „eine Theorie besonderer Praxisinstitutionen - vor allem der Jugendhilfe und Sozialarbeit. " (Böhnisch 1979, S. 22, zit. nach Hamburger 2012, S. 14).

2. Berechtigt ist hier jedenfalls die Frage, ob die Problematik allein an der Zugangsgerechtigkeit zum tertiären Bildungssystem festzumachen ist, oder ob nicht (ergänzend dazu) eine Aufwertung von anderen Berufsgruppen und Bildungsbiografien anvisiert werden müsste? (Vielen Dank an Veronika Reidinger für diese kritische Anmerkung.).

3. An der FH St. Pölten werden gegenwärtig im Rahmen eines Projektes „Weiterbildung Neu“ Modelle zur Anerkennung individueller Bildungsbiografien und Ermöglichung individueller Studienpfade entwickelt. Unsere Überlegungen sind zum großen Teil in Diskussionen im Rahmen des Projektes für das Studium Sozialpädagogik entstanden.

4. Das Bundesgesetz über Fachhochschul-Studiengänge (Fachhochschul-Studiengesetz-FHStG) ,regelt die Durchführung von Fachbochschul-Studiengängen und Lehrgängen zur Weiterbildung sowie die Verleihung der Bezeichnung ,Fachhochschule" in Österreich ( $\mathbb{1} 1 \mathrm{FHStG}$ ). Im $\mathbb{3} 3 \mathrm{FHStG}$ werden, "Ziele und leitende Grundsätze von Fachbochschul-Studiengängen “ formuliert.

5. Zu den Rabmenbedingungen vgl. BMWFW (2017, S. 20 ff.); kritisch dazu vgl. FHK (2016).

\section{Literatur}

BMWFW (2017). Nationale Strategie zur sozialen Dimension in der Hochschulbildung. Für einen integrativeren Zugang und eine breitere Teilhabe. Wien. https://bmbwf.gv.at/fileadmin/user_upload/Nationale_Strategie/_BF_Strategien_kern_gesamt_2_Endversion.pdf. Zugegriffen: 2. Aug. 2019.

FHK (2016). Stellungnahme zum Entwurf der nationalen Strategie zur sozialen Dimension in der Hochschulbildung. Wien. https://www.fhk.ac.at/index.php?eID=tx_nawsecuredl\&u=0\&file=uploads/tx_sbdownloader/FHK_ Stellungnahme_zur_Nationalen_Strategie_zur_sozialen_Dimension.pdf\&t= 1565269084 \&hash=a43e6828838097a624df7d21e9cbfb6188fef7c1. Zugegriffen: 9. Aug. 2019.

Haag, C. (2009). Die Wirklichkeit von Bildung? Erfahrungswissenschaftliche Erkundungen vor dem Hintergrund sozialpädagogischer Bildungsforschung. In S. Neumann \& P. Sandermann (Hrsg.), Kultur und Bildung - Neue Fluchtpunkte für die sozialpädagogische Forschung (S. 215-235). Wiesbaden: VS. 
Hamburger, F. (2012). Einführung in die Sozialpädagogik. Bd. 3. Stuttgart: Kohlhammer.

HSK (2015). Empfehlungen der Hochschulkonferenz zur Förderung nicht-traditioneller Zugänge im gesamten Hochschulsektor. Wien. http:// www.hochschulplan.at/wp-content/uploads/2016/05/Empfehlungender-Hochschulkonferenz-zur-F\%C3\%B6rderung-nicht-traditionellerZug\%C3\%A4nge-im-gesamten-Hochschulsektor_bf.pdf. Zugegriffen: 9. Aug. 2019.

Kallhoff, A. (2012). Das öffentliche Gut in der Reflexion der politischen Ethik. Antrittsvorlesung von Angela Kallhoff im Rahmen des Dies Facultatis an der Universität Wien zum Thema „Bildung als ein öffentliches Gut“ 23.05.2012. https://homepage.univie.ac.at/angela.kallhoff/pdf/Antrittsvorlesung_Wien.pdf. Zugegriffen: 9. Aug. 2019.

Marginson, S. (2012). The problem of public good(s) in higher education. 41st Australian Conference of Economists. Melbourne 12.07.2012, Panel Economic Challenges in Higher Education. https://www.ses.unam.mx/curso2014/pdf/Marginson.pdf. Zugegriffen: 7. Aug. 2019.

Rauschenbach, T., \& Otto, H.-U. (2008). Die neue Bildungsdebatte. Chance oder Risiko für die Kinder- und Jugendhilfe? In T. Rauschenbach $\&$ H.-U. Otto (Hrsg.), Die andere Seite der Bildung. Zum Verhältnis von formellen und informellen Bildungsprozessen (2. Aufl. S. 9-29). Wiesbaden: VS.

Thiersch, H. (2008). Bildung als Projekt der Moderne. In T. Coelen \& H.-U. Otto (Hrsg.), Grundbegriffe Ganztagsbildung. Das Handbuch (S. 977-983). Wiesbaden: VS.

Thole, W., \& Höblich, D. (2008). „Freizeit“ und „Kultur“ als Bildungsorte - Kompetenzerwerb über nicht-formale und informelle Praxen von Kindern und Jugendlichen. In C. Rohlfs, M. Harring \& C. Palentien (Hrsg.), Kompetenz-Bildung. Soziale, emotionale und kommunikative Kompetenzen von Kindern und Jugendlichen (S. 69-93). Wiesbaden: VS.

Tippelt, R., \& Schmidt, B. (2009). Einleitung der Herausgeber. In R. Tippelt \& B. Schmidt (Hrsg.), Handbuch Bildungsforschung (Bd. 2, S. 9-19). Wiesbaden: VS.

Tomic Hensel, M. (2019). Ökonomisierung als Widerspruchsbearbeitung. Kritisch-analytische (Forschungs-)Perspektiven für die Soziale Arbeit. In: soziales_kapital Nr. 21/2019, S. 59-73. https://soziales-kapital.at/index. php/sozialeskapital/article/viewFile/605/1093.pdf. Zugegriffen: 14. Nov. 2019.

Wolter, A. (2013). Massification and Diversity: Has the Expansion of Higher Education Led to a Changing Composition of the Student Body? European and German Experiences. In I. Cerar (Hrsg.), Higher Education Reforms: Looking Back-Looking Forward. 10th International Workshop on Higher Education Reform, Ljubljana. (S. 202-220). https://www.dlib.si/ stream/URN:NBN:SI:DOC-RLHGULW5/0873630c-0256-446f-a987-0ec6922c92f6/PDF [02.08.2019]. 